\title{
Safety and Efficacy of High Power Shorter duration Ablation for Atrial Fibrillation: A Systematic Review and Meta-Analysis.
}

\author{
miao-fu Li ${ }^{1}$, Jing $\mathrm{Wu}^{2}$, Chao-feng $\mathrm{Chen}^{2}$, Mei-jun $\mathrm{Liu}^{2}$, and Yi-zhou $\mathrm{Xu}^{1}$ \\ ${ }^{1}$ Nanjing Medical University \\ ${ }^{2}$ Hangzhou First People's Hospital
}

August 17, 2020

\begin{abstract}
Background: Radiofrequency ablation in patients with atrial fibrillation (AF) is effective but hampered by pulmonary veins reconnection due to insufficient lesions. High power shorter duration ablation (HPSD) seen to increase efficacy and safety. This analysis aimed to evaluate the clinical benefits of HPSD in patients with AF. Methods: The Medline, PubMed, Embase, and the Cochrane Library databases were searched for studies comparing HPSD and Low power longer duration (LPLD) ablation. Results: A total of seven trials with 2023 patients were included in the analysis. Pooled analyses demonstrated that HPSD showed a benefit of first-pass pulmonary vein isolation (PVI) [risk ratio (RR): 1.27; 95\% confidence interval (CI): 1.18-1.37, P $<0.001$ ]. HPSD could reduce recurrence of atrial arrhythmias (RR: 0.70; 95\% CI: 0.50-0.98, P = 0.04). Additionally, HPSD was more beneficial in terms of procedural time [Weighted Mean Difference, (WMD): $-44.62 ; 95 \%$ CI, -63.00 to $-26.23, \mathrm{P}<$ 0.001], ablation time (WMD: -21.25 ; $95 \%$ CI: -25.36 to -17.13 , P < 0.001), and fluoroscopy time (WMD: $-4.13 ; 95 \%$ CI: -7.52 to $-0.74, \mathrm{P}<0.001)$. Moreover, major complications and esophageal thermal injury (ETI) were similar between two groups (RR: 0.75; 95\% CI: $0.44-1.30, \mathrm{P}=0.31$ ) and (RR: $0.64 ; 95 \%$ CI: $0.17-2.39, \mathrm{P}=0.51$ ). Conclusion: HPSD was safe and efficient for treating AF with clear advantages of procedural features, it also showed benefits of higher first-pass PVI and reducing recurrence of atrial arrhythmias compared with the LPLA. Moreover, major complications and ETI were similar between two groups.
\end{abstract}

Safety and Efficacy of High Power Shorter duration Ablation for Atrial Fibrillation: A Systematic Review and Meta-Analysis

\section{Cover page}

Full title: Safety and Efficacy of High Power Shorter duration Ablation for Atrial Fibrillation: A Systematic Review and Meta-Analysis.

Running Title: HPSD ablation for AF treatment.

Word count: 3188 words

All co-authors:

Miao -fu Li M.S. ${ }^{\text {, }}$,Jing-Wu, M.S. ${ }^{\text {b }}$, Chao-feng Chen M.S. ${ }^{\text {b }}$ Mei-jun Liu M.S. ${ }^{\text {b }}$, Yi-zhou Xu M.D ${ }^{\text {a }, b}$.

a Nanjing Medical University, Nanjing, Jiangshu Prov, China.

b Department of Cardiology, Hangzhou First People's Hospital, Hangzhou, Zhejiang Prov, China.

Corresponding Author: Yi-zhou Xu M.D., Department of Cardiology, Nanjing Medical University, Hangzhou First People's Hospital , \#261 Huansha Road, Hangzhou, Zhejiang Province 310000, China. Fax: 8618268805895; E-mail:sci1989@163.com. 
Miao-fu Li , Jing Wu and Chao-feng Chen contributed equally to this article and should be considered equal first authors.

\section{Review criteria}

The Medline, PubMed, Embase, and the Cochrane Library databases were searched for studies comparing high power shorter duration (HPSD) and Low power longer duration (LPLD) ablation for atrial fibrillation treatment. Specific searching strategy was listed in the section of materials and methods.

\section{Message for the clinic}

Pulmonary vein isolation (PVI) is established as the cornerstone for treating atrial fibrillation (AF). However, the low power longer duration (LPLD) ablation always accompanied by a higher recurrence and the clinical benefits of high power shorter duration (HPSD) ablation were inconsistent. In our analysis, we found that the approach of HPSD seen to be effective and safe for AF treatment.

\section{Summary}

Background: Radiofrequency ablation in patients with atrial fibrillation (AF) is effective but hampered by pulmonary veins reconnection due to insufficient lesions. High power shorter duration ablation (HPSD) seen to increase efficacy and safety. This analysis aimed to evaluate the clinical benefits of HPSD in patients with AF.

Methods: The Medline, PubMed, Embase, and the Cochrane Library databases were searched for studies comparing HPSD and Low power longer duration (LPLD) ablation.

Results: A total of seven trials with 2023 patients were included in the analysis. Pooled analyses demonstrated that HPSD showed a benefit of first-pass pulmonary vein isolation (PVI) [risk ratio (RR): 1.27; 95\% confidence interval (CI): 1.18-1.37, P < 0.001]. HPSD could reduce recurrence of atrial arrhythmias (RR: $0.70 ; 95 \%$ CI: $0.50-0.98, \mathrm{P}=0.04)$. Additionally, HPSD was more beneficial in terms of procedural time [Weighted Mean Difference, (WMD): $-44.62 ; 95 \%$ CI, -63.00 to $-26.23, \mathrm{P}<0.001$ ], ablation time (WMD: $-21.25 ; 95 \%$ CI: -25.36 to $-17.13, \mathrm{P}<0.001$ ), and fluoroscopy time (WMD: -4.13 ; 95\% CI: -7.52 to -0.74 , $\mathrm{P}<0.001$ ). Moreover, major complications and esophageal thermal injury (ETI) were similar between two groups (RR: $0.75 ; 95 \% \mathrm{CI}: 0.44-1.30, \mathrm{P}=0.31$ ) and (RR: $0.64 ; 95 \% \mathrm{CI}: 0.17-2.39, \mathrm{P}=0.51$ ).

Conclusion: HPSD was safe and efficient for treating AF with clear advantages of procedural features, it also showed benefits of higher first-pass PVI and reducing recurrence of atrial arrhythmias compared with the LPLA. Moreover, major complications and ETI were similar between two groups.

Key words: Atrial fibrillation; Low power longer duration ablation; High power shorter duration ablation; Safety, Efficacy.

\section{INTRODUCTION}

Pulmonary vein isolation (PVI) is established as the cornerstone for treating AF ${ }^{1}$. Efficacy of PVI is associated with trans-mural, continuous, permanent cellular damage ${ }^{2}$. Multiple factors outside of power can impact energy delivery to the tissue and total energy delivered may be amplified or attenuated depending on contact force $(\mathrm{CF})$ and catheter stability ${ }^{3,4}$. Despite the technical advances with force sensing and stability monitoring, the rate of PVs reconnection remains frequently with Low power longer duration (LPLD) $\left(20^{\sim} 40 \mathrm{~W}\right.$, with longer ablation duration of $10-30 \mathrm{~s}$ per site). Because retaining catheter stability in a constantly working heart for a long time is very difficult, and tissue edema by long duration ablation limits effective ablation ${ }^{2}$. Moreover, the left atrium is adjacent to the esophagus, injury depth may be excessive by long duration ablation despite a lower power setting, thus increasing the risk of esophageal thermal injury (ETI) ${ }^{5}$. Simultaneously, a large fluid volume infused via a catheter during ablation, which is proportional to the ablation time, can induce acute heart failure in patients with impaired left ventricular function ${ }^{6}$. In order to optimize AF ablation, a novel energy delivery strategy with high power shorter duration (HPSD) 
ablation was applied for AF treatment ${ }^{7}$. To create similar lesions, the ablation time is longer by LPLD than HPSD ablation.

HPSD ablation has been supposed to increase efficacy and minimize deep tissue injury. However, studies comparing data between the HPSD and LPLD approaches were limited and inconsistent. Therefore, this systematic review and meta-analysis was performed to evaluate the efficacy and safety of HPSD compared with LPLD in treating AF.

\section{MATERIALS AND METHOD}

\subsection{Search strategy}

Relevant studies were searched in the Medline, PubMed, Embase, the Cochrane Library, and Elsevier's ScienceDirect databases. Reports published in non-English languages were excluded from the search. The search strategy employed the following relevant key words and medical subject heading terms: Atrial fibrillation; AF; Radiofrequency; RF; Catheter ablation; High power; High-power; High-power shorter-duration; HPSD; Low power; Low-power long duration; LPLD and conventional power. The literature search was updated in August 12, 2020.

\subsection{Inclusion and exclusion criteria}

Two reviewers (C-CF and J-W) screened and identified studies that met the following inclusion criteria: (1) patients with drug-refractory symptomatic AF who accepted radiofrequency ablation; (2) wide-area circumferential ablation (WACA) for PVI applied using irrigated-tip catheters; (3) patients undergoing treatment using CA for the first time; (4) comparison between HPSD and LPLD approaches; (5) sample size [?]20; and (6) studies needed to provide at least one of the reliable information with regard procedure outcomes, complications, first-pass PVI, acute PVs reconnection, and follow-up in both groups. The exclusion criteria were as follows: (1) an equivocal study design or group allocation; and (2) conference abstracts, case reports, case series studies, editorials, review articles, or non-English language articles.

\subsection{Study selection}

Two reviewers (L-MF, J-W.) independently screened the titles and abstracts and excluded articles that were irrelevant to the topic. The reviewers then evaluated the full text of eligible articles for suitability based on the strict inclusion and exclusion criteria. A third reviewer (C-CF.) was used to resolve discrepancies.

\subsection{Quality assessment and data extraction}

The study quality was evaluated by two investigators (L-MF and L-MJ) using the Newcastle-Ottawa Scale (NOS) for observational studies and Delphi consensus criteria for randomized controlled study (RCTs). The NOS system consisted of eight questions with nine possible points. A star system was used to judge the data according to the selected populations and the comparability of the groups and exposure/outcome of interest. A study with NOS [?]7 was judged to be a study of good quality ${ }^{8}$. The Preferred Reporting Items for Systematic Reviews and Meta-analyses Amendment to the Quality of Reporting of Meta-analyses Statement and recommendations from the Cochrane Collaboration in epidemiology were followed while performing the present systematic review. Data extraction was conducted by mutual agreement, and all potential disagreements were solved by consensus ${ }^{9,10}$.

\subsection{Outcomes definitions}

HPSD: Ablation power $>40 \mathrm{~W}$, with shorter duration of $2 \sim 10$ s per site.

LPLD: Ablation power limited to 20 40W, with longer ablation duration of 10-30 s per site.

Procedure time: It is the time from the application of local anesthesia to the withdrawal of all catheters.

Ablation time: It is the time from the first to the last application.

Fluoroscopy time: It is the time of fluoroscopy from the start to the end of the procedure. 
Atrial arrhythmias recrudescence: It is any symptomatic or asymptomatic atrial arrhythmia lasting $>30$ seconds after completing the blanking period after catheter ablation.

First-pass PVI: Rate of complete PVI after first pass circumferential radiofrequency delivery.

Major complications: It is defined as complications that required any intervention or prolonged hospital stay.

ETI: Endoscopy or MRI image were performed to assess esophageal thermal injury post-ablation.

\subsection{Statistical analysis}

A meta-analysis of the summary statistics from individual trials was performed. The statistical analysis was completed by an independent statistician (C-CF). The differences in dichotomous variables and outcome endpoints were reported as an odds ratio (OR) or risk ratio (RR) with $95 \%$ confidence intervals (CIs). The continuous variables were analyzed using weighted mean differences (WMD) or standard mean differences (SMD). Fixed- and random-effects models used weighting based on an inverse variance, which was calculated according to DerSimonian and Laird ${ }^{11}$. The between-study heterogeneity was reflected by $I^{2}>50 \%$, with a $P<0.05$ deemed statistically significant. In cases of statistical heterogeneity, sensitivity analyses were performed to assess the contribution of each study to the pooled estimate by sequentially excluding individual trials and recalculating the pooled RR estimate for the remaining studies ${ }^{12}$. When the pooled analysis still yielded significant heterogeneity, the random-effects model was used. The statistical analysis was performed using the Review Manager (version 5.3; Copenhagen : The Nordic Cochrane Center, The Cochrane Collaboration, 2014).

\section{RESULTS}

\subsection{Study and data selection}

The flowchart of the detailed search process is illustrated in Figure 1. Initially, 397 potentially studies were identified, of which 73 were duplicates and 233 were excluded after reviewing the titles and abstracts. Of the remaining 91 studies, 17 review articles, 3 editorial/letters, and 8 case reports or case series, and 21 abstracts were excluded. Further, 33 studies were excluded after a detailed evaluation of the full text for the following reasons: 9 were uncontrolled studies, 5 were clinical studies design, 13 lacked study endpoints, 6 reporting duplicate data. And then, 2 trails by Berte B et al and Dhillon G et al ${ }^{15,16}$ comparing HPSD vs. LPLD were also excluded, because their ablation were guided by ablation index (AI) without illustrating ablation time per site detailed. Consequently, 7 clinical trials with 2023 patients were enrolled in this meta-analysis $4,13,14,17-20$.

\subsection{Study characteristics}

The characteristics of the included trials and ablation settings are shown in Table 1. A significant difference in power delivery setting was found between the HPSD and LPLD groups. There is no consensus about the power and duration settings for HPSD, in our study, energy levels above $40 \mathrm{~W}$ are considered as high power and duration of application for $2^{\sim} 10$ s as short duration ${ }^{4,13,14,17-20}$. All patients received PVI, additional linear ablations were performed in select patients at the operator's discretion in some trials ${ }^{4,18,19}$. All studies had good methodological quality. The results of the grouping ensured the feasibility of this metaanalysis.

\subsection{Clinical outcomes}

In all included studies, the HPSD approach was found to be associated with a high rate of first-pass PVI (RR: 1.28; 95\% CI: 1.12-1.46, P < 0.001 Fig 2A). Additionally, after a mean follow-up of 16 months, the pooled analysis indicated that the HPSD could reduce the recurrence of atrial arrhythmia (OR: 0.70; 95\% CI: $0.50-0.98, \mathrm{P}=0.04 ;$ Fig $2 \mathrm{~B})$. Moreover, the HPSD approach could significantly reduce the total procedural time (WMD: $-44.62 ; 95 \%$ CI, -63.00 to -26.23 , P < 0.001; Fig. 3A), ablation time (WMD: $-21.25 ; 95 \%$ CI: -25.36 to $-17.13, \mathrm{P}<0.001$; Fig. 3B), and fluoroscopy time (WMD: $-4.13 ; 95 \%$ CI: -7.52 to $-0.74, \mathrm{P}<0.001$; 
Fig. 3C). Major complications and ETI were similar between two groups (RR: 0.75; 95\% CI: 0.44-1.30, P $=0.31$; Fig.2C) and (RR: 0.64; 95\% CI: 0.17-2.39, $\mathrm{P}=0.51$ Fig. 2D).

\section{DISCUSSION}

The main findings were as follows: (1) HPSD approach was associated with higher first-pass PVI, and recurrence of atrial arrhythmias; (2) HPSD approach could significantly reduce procedural time, ablation time and fluoroscopy time compared with the LPLD approach; and (3) Major complications and ETI were similar between two groups.

Thermal injury by RF ablation comprises two consecutive phases: resistive and conductive. The balance between power and duration parameters in resistive and conductive heating has a significant influence on lesion creation. The resistive phase has a resistive component adjacent to the catheter tip, which results in local heating and dissipation of energy as a heat source. Resistive heating probably occurs in the first few seconds during the RF application. With immediate heating, the electrical current is delivered at the catheter-tissue interface. The tissue necrosis is confined only to the first 1-1.5 mm from the catheter tip; however, the temperature always rises above $50^{\circ} \mathrm{C}$ with a conventional power setting ${ }^{17}$. Greater resistive heating can be achieved using higher-power delivery. In the conductive phase, the resistive heat source then extends energy passively to deeper tissues. Conductive heating is time dependent. The heating of deeper tissues increases with longer-duration RF applications ${ }^{2,7,21-23}$. The LPLD approach is associated with longer conduction heating.

Low power longer duration time of 10-30 s per site is based on earlierin vivo studies on the ventricular tissue 7 . Simmers et al. showed that the lesion dimensions increased in $30 \mathrm{~s}$ using $25 \mathrm{~W}$ power; however, the mean lesion depth already reached $7.25 \mathrm{~mm}$ in $30 \mathrm{~s}^{24}$. In the left atrium, where the mean posterior wall thickness is $1.5-2.5 \mathrm{~mm}$ and the esophageal distance from the posterior wall is as small as $2.5 \mathrm{~mm}$, the LPLD approach might cause serious injury to the esophagus $7,18,24,25$. The incidence of atrio-esophageal fistula (AEF) is reported as $0.1 \%-0.25 \%$, and the incidence of esophageal thermal injury (ETI) is $2 \%-50 \%$ for the LPLD approach $^{18}$. In addition, longer procedural time, ablation time, and fluoroscopy time are necessary for the LPLD approach due to its inherent characteristics. Prolonged procedural time would inevitably lead to a longer anesthesia duration, which also increases the procedural risk, especially for elderly patients ${ }^{26}$.

One potential approach to optimize the longer ablation time of LPLD is to modify the relationship between the resistive and the conductive heating phases by increasing the resistive heating phase and reducing the conductive hearting phase to deliver immediate heating to the full thickness of tissues and limit deep tissue injury $2,17,27$. To achieve this, high energy must be delivered in a short duration. Thus, the strategy of HPSD was proposed and applied for AF ablation.

The HPSD approach is largely based on immediate heating during the resistive phase, however, whether high power with shorter duration might create trans-mural lesions in the left atrium? The average thickness of the left atrium is $2.8 \pm 1.1 \mathrm{~mm}$ in humans ${ }^{28}$. For patients with persistent AF, the mean atrial wall thickness is only $1.89 \pm 0.5 \mathrm{~mm}$ and never exceeds $3.5 \mathrm{~mm}^{25}$. The HPSD approach affects a tissue depth of $3.5-4 \mathrm{~mm}^{2}$, thus, the left atrial wall thickness is well within the depth for the HPSD approach. Hence, HPSD is well suited to AF ablation. Several previous experiments evaluated the utility of HPSD approach. Bourier et al evaluated the lesion metrics created by HPSD compared with LPLD application. They found that the HPSD approach created lesions similar in volume but wider and shallower compared with the LPLD approach ${ }^{29}$. In fresh killed porcine ventricles, Goyal et al. showed that for a given CF, 20 s were needed to create a 4 $\mathrm{mm}$ deep lesion using $20 \mathrm{~W}$ ablation, while only $6-7$ s were enough for $50 \mathrm{~W}$ ablation ${ }^{22}$. Bhaskaran et al. examined several combinations of high-power ablation in vitro and in a sheep model. The study showed that the use of $50 \mathrm{~W}$ for $5 \mathrm{~s}$ resulted in a similar lesion depth compared with the use of $40 \mathrm{~W}$ for $30 \mathrm{~s}$. Steam pops occurred in $8 \%$ of the $40 \mathrm{~W}(30 \mathrm{~s})$ ablation and in none of the $50 \mathrm{~W}(5 \mathrm{~s})$ ablation ${ }^{7}$. So, HPSD approach could achieve rapid, more controlled, resistive tissue heating, and avoiding deeper collateral injury.

For clinical studies, Winkle et al showed that HPSD approach using CF sensing catheter was safe and result in excellent long-term freedom from $\mathrm{AF}$ with short procedure times and delivery of small amounts of total 
RF energy. No complications were reported in this trial ${ }^{27}$. Other studies also showed the safety and efficacy of HPSD approach in an abstract form. Nonetheless, these studies are too small to evaluate the safety and efficacy of HPSD approach, and the comparison data between HPSD and LPLD were still limited. So, the present meta-analysis was performed.

The present study showed a higher first-pass PVI in the HPSD group when compared to the LPLD group. The main reasons may due to greater size, more uniform and better consistency of the lesions created by HPSD approach. Catheter-tissue contact stability is an important factor contributing to lesion creation, and catheter instability in a constantly moving heart may account for the difficulty to transmit heat to the tissue $^{17}$. This shortening of HPSD approach may mitigate the negative effects of catheter instability and probably optimizes lesion creation by increasing the likelihood to keep the catheter stable throughout the entire application, before stability becomes a consideration ${ }^{27}$. In the LPLD group, the catheter stability was an issue when longer single-lesion ablation was needed, leading to unevenness of lesions, tissue edema, and lower rate of first-pass PVI. Moreover, HPSD approach could reduce the rate of long-term recurrence of atrial arrhythmias. Complete PVI with trans-mural injury is most important for the freedom from AF during the long-term follow-up ${ }^{30,31}$. Hence, HPSD approach trend to form more trans-mural, continuous and permanent lesions.

The pooled analysis showed significant advantages of the HPSD approach. The approach could shorten procedural time and ablation time compared with the LPLD approach, thus limiting patient exposure to intravenous fluids and anesthesia. Additionally, fluoroscopy time was also shorter in the HPSD group, which had a direct favorable impact on the patient, operator, and supporting staff. These results were consistent with previously published findings $4,16,20,22,23,32$. A significant reduction in the procedural time was observed in the HPSD group because of shorter ablation time. The shorter ablation time for HPSD were due to the shorter time required for lesion creation, higher first-pass PVI, and fewer acute PV reconnections ${ }^{2}$. In the LPLD group, additional ablations were required for gap ablation in non-transmural lesions and achieving biphasic block of PVs so as to achieve completed PVI ${ }^{17}$. During the procedure, catheter movement mostly relies on the combination of x-ray and 3D electro-anatomic mapping system. Hence, longer ablation time in the LPLD group inevitably led to greater fluoroscopy time to locate and move the catheter in the left atrium. Additionally, less irrigation fluid was needed during HPSD due to shorter ablation time, making HPSD more suitable for patients with impaired left ventricular function.

Previous studies involved an increased power of 40-50 W for AF ablation. One study using HPSD showed improved outcomes but an increase in complications, such as ETI, cardiac tamponade, and so forth. The safety of using high power for AF ablation, especially on the posterior wall, was a concern ${ }^{33}$. However, another study using HPSD ablation reported no increase in complications ${ }^{34}$. According to the principle of HPSD with increasing resistive and reducing conductive phases, minimizing damage to collateral tissues has been a crucial consideration in HPSD. Several animal studies suggested that HPSD was superior to LPLD with lower complication rate ${ }^{2,21}$. Some human studies using HPSD showed excellent clinical outcomes with fewer complications ${ }^{15,35,36}$. In the present study, the pooled analysis of included studies also showed that the rate of complications similar between two groups. The AEF or ETI was rarely reported because most of the studies were single-center studies, and none of them were large enough to evaluate infrequent serious complications. A large observational study focused on the complications of the HPSD approach. In this study, 13,974 ablations were performed on 10,284 patients, revealing an extremely low complication rate in the HPSD group. Only 1 AEF occurred in 11,436 ablations using HPSD; however, 3 AEFs occurred in 2538 ablations using LPLD ${ }^{32}$. There were two studies included in our analysis discussed ETI in patients received ablation treatment. The results showed low and similar rates of ETI in the two groups. The subgroup analysis showed that HPSD could reduce mild ETI. No AEFs were reported in the included studies. Thus, the HPSD approach was safe enough for AF ablation ${ }^{18}$.

Additionally, another study reported by Reddy et al compared very high power short-duration (vHPSD, a delivery of $90 \mathrm{~W}$ for $4 \mathrm{~s}$ ) approach using a QDOT microcatheter (Biosense Webster, Inc., CA, USA) with the LPLD approach. They demonstrated that vHPSD was an efficient, feasible, and safe strategy for AF 
ablation. However, this study was not included in the present meta-analysis due to a completely different setting of delivery of the vHPSD and HPSD approaches. Nevertheless, vHPSD may be another promising strategy in the future ${ }^{23}$.

Our study has some limitations. First, publication bias could not be completely excluded, the inclusion of only published data contributed to bias. Second, the number of included studies was limited to only seven, and most of the studies were designed as nonrandomized, thus, more well-designed and large-scale RCTs are required to confirm the findings. Third, limited collateral tissue damage is one of the important advantages of HPSD. However, in the present meta-analysis, this damage was not completely reflected due to limited endpoints reported from the included studies. Fourth, in some studies, the catheters applied in the HPSD and LPLD groups were different, thus may affect the outcomes of the pooled analysis. Fifth, the ablation power and duration settings in the included studies were not completely consistent.

\section{COCLUSIONS}

The present systematic review and meta-analysis demonstrated that HPSD was a feasible, efficient, safe, and effective approach for AF ablation. The approach had some clear advantages over the LPLD approach, including reduced procedure time, ablation time, and fluoroscopy time. Simultaneously, the HPSD approach was associated with higher first-pass PVI compared with the LPLD approach. Additionally, the HPSD approach could reduce the recurrence of atrial arrhythmias. Moreover, HPSD was as safe as LPLD with low rate of complications. However, there is no consensus about the power and duration settings for HPSD. Therefore, more clinical trials should be conducted to optimize dwell times, power setting, and even catheter selection so as to consistently create perfect lesions in the atrium.

\section{REFERENCES}

1. Chen CF, Gao XF, Duan X, et al. Comparison of catheter ablation for paroxysmal atrial fibrillation between cryoballoon and radiofrequency: A meta-analysis. J Interv Card Electrophysiol. 2017;48:351-366.

2. Leshem E, Zilberman I, Tschabrunn CM, et al. High-power and short-duration ablation for pulmonary vein isolation: Biophysical characterization. JACC Clin Electrophysiol. 2018;4:467-479.

3. Patel PJ, Padanilam BJ. High-power short-duration ablation: Better, safer, and faster? J Cardiovasc Electrophysiol. 2018;29:1576-1577.

4. Bunch TJ, May HT, Bair TL, et al. Long-term outcomes after low power, slower movement versus high power, faster movement irrigated-tip catheter ablation for atrial fibrillation. Heart Rhythm.2020 Feb;17(2):184-189.

5. Thiyagarajah A, Kadhim K, Lau DH, et al. Feasibility, safety, and efficacy of posterior wall isolation during atrial fibrillation ablation: A systematic review and meta-analysis.Circ Arrhythm Electrophysiol.2019 Aug;12(8):e007005.

6. Solimene F, Lepillier A, De Ruvo E, et al. Reproducibility of acute pulmonary vein isolation guided by the ablation index. Pacing Clin Electrophysiol. 2019 Jul;42(7):874-881.

7. Bhaskaran A, Chik W, Pouliopoulos J, et al. Five seconds of 50-60 w radio frequency atrial ablations were transmural and safe: An in vitro mechanistic assessment and force-controlled in vivo validation. Europace. 2017;19:874-880.

8. Stang A. Critical evaluation of the newcastle-ottawa scale for the assessment of the quality of nonrandomized studies in meta-analyses. Eur J Epidemiol. 2010 Sep;25(9):603-5.

9. Stroup DF, Berlin JA, Morton SC, et al. Meta-analysis of observational studies in epidemiology: A proposal for reporting. Meta-analysis of observational studies in epidemiology (moose) group. JAMA. 2000 Apr 19;283(15):2008-12.

10. Moher D, Cook DJ, Eastwood S, et al. Improving the quality of reports of meta-analyses of randomised 
controlled trials: The quorom statement. Onkologie. 2000 Dec;23(6):597-602.

11. DerSimonian R, Laird N. Meta-analysis in clinical trials. Controlled clinical trials. 1986;7:177-188.

12. Bowden J, Tierney JF, Copas AJ, et al. Quantifying, displaying and accounting for heterogeneity in the meta-analysis of rcts using standard and generalised q statistics. BMC Med Res Methodol. 2011 Apr 7;11:41.

13. Castrejón-Castrejón S, Martínez Cossiani M, Ortega Molina M, et al. Feasibility and safety of pulmonary vein isolation by high-power short-duration radiofrequency application: Short-term results of the power-fast pilot study. J Interv Card Electrophysiol.2020 Jan;57(1):57-65.

14. Kottmaier M, Popa M, Bourier F, et al. Safety and outcome of very high-power short-duration ablation using $70 \mathrm{w}$ for pulmonary vein isolation in patients with paroxysmal atrial fibrillation. Europace. 2020;22:388393.

15. Berte B, Hilfiker G, Russi I, et al. Pulmonary vein isolation using a higher power shorter duration close protocol with a surround flow ablation catheter. J Cardiovasc Electrophysiol. 2019 Nov;30(11):2199-2204.

16. Dhillon G, Ahsan S, Honarbakhsh S, et al. A multicentered evaluation of ablation at higher power guided by ablation index: Establishing ablation targets for pulmonary vein isolation. J Cardiovasc Electrophysiol. 2019;30:357-365.

17. Pambrun T, Durand C, Constantin M, et al. High-power (40-50 w) radiofrequency ablation guided by unipolar signal modification for pulmonary vein isolation. Circ Arrhythm Electrophysiol. 2019 Jun;12(6):e007304.

18. Baher A, Kheirkhahan M, Rechenmacher SJ, et al. High-power radiofrequency catheter ablation of atrial fibrillation: Using late gadolinium enhancement magnetic resonance imaging as a novel index of esophageal injury. JACC. Clinical electrophysiology. 2018;4:1583-1594.

19. Yazaki K, Ejima K. Impedance drop predicts acute electrical reconnection of the pulmonary vein-left atrium after pulmonary vein isolation using short-duration high-power exposure. J Interv Card Electrophysiol. 2020 Jan 4.

20. Vassallo F, Cunha C, Serpa E, et al. Comparison of high-power short-duration (hpsd) ablation of atrial fibrillation using a contact force-sensing catheter and conventional technique: Initial results. J Cardiovasc Electrophysiol. 2019 Oct;30(10):1877-1883.

21. Barkagan M, Contreras-Valdes FM, Leshem E, et al. High-power and short-duration ablation for pulmonary vein isolation: Safety, efficacy, and long-term durability. J Cardiovasc Electrophysiol. 2018 Sep;29(9):12871296.

22. Ali-Ahmed F, Goyal V, Patel M, et al. High-power, low-flow, short-ablation duration-the key to avoid collateral injury? J Interv Card Electrophysiol. 2019;55:9-16. doi: 10.1007/s10840-018-0473-5.

23. Reddy VY, Grimaldi M, De Potter T, et al. Pulmonary vein isolation with very high power, short duration, temperature-controlled lesions: The qdot-fast trial. JACC: Clinical Electrophysiology. 2019;5:778-786.

24. Simmers TA, Tukkie R. How to perform pulmonary vein isolation for the treatment of atrial fibrillation: Use of the localisa catheter navigation system. Europace. 2004;6:92-96.

25. Beinart R, Abbara S, Blum A, et al. Left atrial wall thickness variability measured by ct scans in patients undergoing pulmonary vein isolation. J Cardiovasc Electrophysiol. 2011;22:1232-1236.

26. Jilek C, Lewalter T. Ablation for atrial fibrillation in the elderly. Herz. 2017;28:39-47.

27. Winkle RA, Moskovitz R, Hardwin Mead R, et al. Atrial fibrillation ablation using very short duration $50 \mathrm{w}$ ablations and contact force sensing catheters. J Interv Card Electrophysiol 2018;52:1-8. 
28. Cabrera JA, Ho SY, Climent V, et al. The architecture of the left lateral atrial wall: A particular anatomic region with implications for ablation of atrial fibrillation. European heart journal. 2008;29:356-362.

29. Bourier F, Duchateau J. High-power short-duration versus standard radiofrequency ablation: Insights on lesion metrics. J Cardiovasc Electrophysiol. 2018 Nov;29(11):1570-1575.

30. Andrade JG, Champagne J, Dubuc M, et al. Cryoballoon or radiofrequency ablation for atrial fibrillation assessed by continuous monitoring: A randomized clinical trial. Circulation. 2019 Nov 26;140(22):1779-1788.

31. Mujovic N, Marinkovic M, Lenarczyk R, et al. Catheter ablation of atrial fibrillation: An overview for clinicians. Advances in therapy. 2017;34:1897-1917.

32. Winkle RA, Mohanty S, Patrawala RA, et al. Low complication rates using high power (45-50 w) for short duration for atrial fibrillation ablations. Heart rhythm. 2019;16:165-169.

33. Kanj MH, Wazni O, Fahmy T, et al. Pulmonary vein antral isolation using an open irrigation ablation catheter for the treatment of atrial fibrillation: A randomized pilot study. J Am Coll Cardiol. 2007 Apr 17;49(15):1634-1641.

34. Winkle RA, Mead RH, Engel G, et al. Atrial fibrillation ablation: "Perpetual motion" of open irrigated tip catheters at $50 \mathrm{w}$ is safe and improves outcomes. Pacing Clin Electrophysiol. 2011;34:531-539.

35. Wolf M, El Haddad M, De Wilde V, et al. Endoscopic evaluation of the esophagus after catheter ablation of atrial fibrillation using contiguous and optimized radiofrequency applications. Heart rhythm. 2019;16:10131020 .

36. Plenge T, van den Bruck JH, Luker J, et al. Porous tip contact force-sensing catheters for pulmonary vein isolation: Performance in a clinical routine setting. J Interv Card Electrophysiol. 2020 Mar;57(2):251-259.

\section{DISCLOSURE}

FUNDING: Chao-feng Chen is supported by Hangzhou Science and Technology Sanitation Project (2016A02).

CONFLICTS OF INTEREST: The authors have no conflict of interest to declare.

ETHICS: There were not any ethics problems in our paper.

ACKNOWLEDG EMENTS: Miao-fu Li and Jing Wu: contributed to write the manuscript; Chao-feng Chen contributed to design of this work, statistical analysis and write article; Mei-jun Liu: contributed to evaluate quality and retrieved the required data, Chao-feng Chen: contributed to review the literature, performed the selection of the studies and helped gather references for the manuscript. Dr Yi-zhou Xu: contributed to design of this work, revised and approved the final version of the manuscript.

\section{FIGURE LEGENDS:}

Fig 1: Flow diagram of study selection process.

Fig 2: Forest plots of first-pass PVI of PVs (A), recurrence of atrial arrhythmias (B), major complications (C) and ETI (D) for HPSD vs. LPLD. HPSD: high power shorter duration; LPLD: Low power longer duration; PVI: pulmonary vein isolation; PVs: pulmonary veins, ETI: esophageal thermal injury.

Fig 3: Forest plots of procedural time (A), ablation time (B), fluoroscopy time (C) for HPSD vs. LPLD. HPSD: high power shorter duration; LPLD: Low power longer duration. 
figures/Figure1/Figure1-eps-converted-to.pdf 
figures/Figure2/Figure2-eps-converted-to.pdf 
figures/Figure3/Figure3-eps-converted-to.pdf

\section{Hosted file}

Table 1.docx available at https://authorea.com/users/334009/articles/475762-safety-andefficacy-of-high-power-shorter-duration-ablation-for-atrial-fibrillation-a-systematicreview-and-meta-analysis 